\title{
Evaluation of Inferior Capsular Laxity in Patients with Atraumatic Multidirectional Shoulder Instability with Magnetic Resonance Arthrography
}

\author{
Kyoung-Jin Park, MD, PhD ${ }^{1}$, Ho-Seung Jeong, MD ${ }^{2}$, Ji-Kang Park, MD, PhD'², \\ Jung-Kwon Cha, $\mathrm{MD}^{2}$, Sang-Woo Kang, $\mathrm{MD}^{2}$ \\ ${ }^{1}$ Goodsam Orthopaedic Clinic, Cheongju, Korea; ${ }^{2}$ Department of Orthopaedic Surgery, Chungbuk National University Hospital, Cheongju, Korea
}

Objective: To compare inferior capsular redundancy by using magnetic resonance arthrography (MRA) images in patients with multidirectional instability (MDI) of the shoulder and control subjects without instability and thereby develop a screening method to identify the presence of shoulder MDI.

Materials and Methods: The MRA images of patients with MDI of the shoulder ( $n=65,57$ men, 8 women; mean age, 24.5 years; age range, 18-42 years) treated over an eight-year period were retrospectively reviewed; a control group $(n=65,57$ men, 8 women; mean age, 27.4 years; age range, 18-45 years) without instability was also selected. The inferior capsular redundancy was measured using a new method we named the glenocapsular $(\mathrm{GC})$ ratio method. MRA images of both groups were randomly mixed together, and two orthopedic surgeon reviewers measured the cross-sectional areas (CSAs) and sagittal capsule-head ratios on oblique sagittal images, as well as the axial capsule-head ratios on axial images and GC ratios on oblique coronal images.

Results: The CSAs and GC ratios were significantly higher in patients than in controls (both, $p<0.001$ ); however, the sagittal capsule-head ratios and axial capsule-head ratios were not significantly different ( $p=0.317, p=0.053$, respectively). In addition, GC ratios determined the presence of MDI more sensitively and specifically than did CSAs. A GC ratio of $>1.42$ was found to be most suggestive of MDI of the shoulder, owing to its high sensitivity (92.3\%) and specificity (89.2\%). Conclusion: $\mathrm{GC}$ ratio can be easily measured and used to accurately screen for MDI of the shoulder.

Keywords: Multidirectional shoulder instability; MDI; Magnetic resonance arthrography; MRA; Capsular redundancy; Inferior instability

\section{INTRODUCTION}

Shoulder multidirectional instability (MDI) was first reported by Neer and Foster (1). They attributed the condition to excessive capsular redundancy and showed that its pathology differed from that of traumatic instability.

Received August 9, 2018; accepted after revision February 13, 2019.

Corresponding author: Ho-Seung Jeong, MD, Department of Orthopaedic Surgery, Chungbuk National University Hospital, 776 1sunhwan-ro, Seowon-gu, Cheongju 28644, Korea.

- Tel: (8243) 269-6077 - Fax: (8243) 269-6359

- E-mail: hoseung1@gmail.com

This is an Open Access article distributed under the terms of the Creative Commons Attribution Non-Commercial License (https://creativecommons.org/licenses/by-nc/4.0) which permits unrestricted non-commercial use, distribution, and reproduction in any medium, provided the original work is properly cited.
MDI was defined as instability in two or three directions (1-6). Neer and Foster (1) highlighted the sulcus sign as the most important indicator of MDI. Patients with MDI show uncontrolled and involuntary inferior subluxation or dislocation associated with anterior and posterior dislocations or subluxations of the shoulder (1). Capsule redundancy is a key predisposing factor in the diagnosis of MDI. Magnetic resonance arthrography (MRA) shows an increased joint capsular volume in MDI $(7,8)$. However, few studies have accurately measured capsular area enlargement in patients with MDI, and there has been little information that can be used to accurately quantify the capsular area in the setting of MDI of the shoulder.

The common components of capsular redundancy in MDI are an elongated inferior capsule and a deficient rotator interval (9-11). These two factors are important for inferior 
stability, and their redundancy is responsible for the sulcus sign observed in MDI patients. A small number of reports have compared MDI patients and non-MDI controls by measuring their rotator interval lengths or calculating their glenoid size versus rotator interval length ratios $(11,12)$, and few studies have addressed the inferior capsule. Lee et al. (11) and Lim et al. (13) measured inferior capsule lengths and showed significant differences between them in patients and in controls. However, these studies involved absolute evaluations that did not consider the sizes of the patient joint structures. The purpose of this study was to identify MDI of the shoulder using glenocapsular (GC) ratios, which were defined as the inferior capsule length divided by glenoid lengths, in order to control for any patient-specific differences. We hypothesized that GC ratios would be significantly higher for MDI patients and controls, and that GC ratios would more accurately detect the presence of MDI compared with any of the previously described methods.

\section{MATERIALS AND METHODS}

\section{Patient Selection}

Our Institutional Review Board approved this retrospective study and waived the requirement for informed consent for the patient data review. Ultimately, 81 patients with MDI of the shoulder were treated during the eight-year period (February 2010-January 2017).
In the patient group, MDI of the shoulder was diagnosed by an orthopedic surgeon with 15 years of experience, based on the findings of physical examination under general anesthesia and arthroscopic surgical findings (Fig. 1). Sixty-five patients ( 57 men, 8 women; mean age, 24.5 years; age range, 18-42 years) constituted the MDI group. The mean time to surgery after an initial instability event was 31 months (10-56 months). All 65 patients had an atraumatic onset as well as positive test results for the following; sulcus sign, anterior and posterior drawer test, and jerk test under general anesthesia. In addition, all patients had general ligament laxity, as indicated by elbow or metacarpophalangeal joint hyperextension, genu recurvatum, and the ability to rest their thumb on their ipsilateral forearm. Patients underwent arthroscopic stabilization surgery following MRA. Notable pathological findings of MDI of the shoulder, including the stadium sign and inferior capsular laxity, were identified during arthroscopy. Patients with bony deficiency (glenoid bone loss $>20 \%$ ), degenerative arthritis, rotator cuff tear, traumatic instability, or a dislocated or subluxated long head biceps tendon on MRA were excluded.

The control group included patients who had normal shoulders on MRA (36 shoulders), those with rotator cuff tendinitis ( 55 shoulders), and those who had a partialthickness rotator cuff tear that involved less than half of the thickness of the supraspinatus or infraspinatus

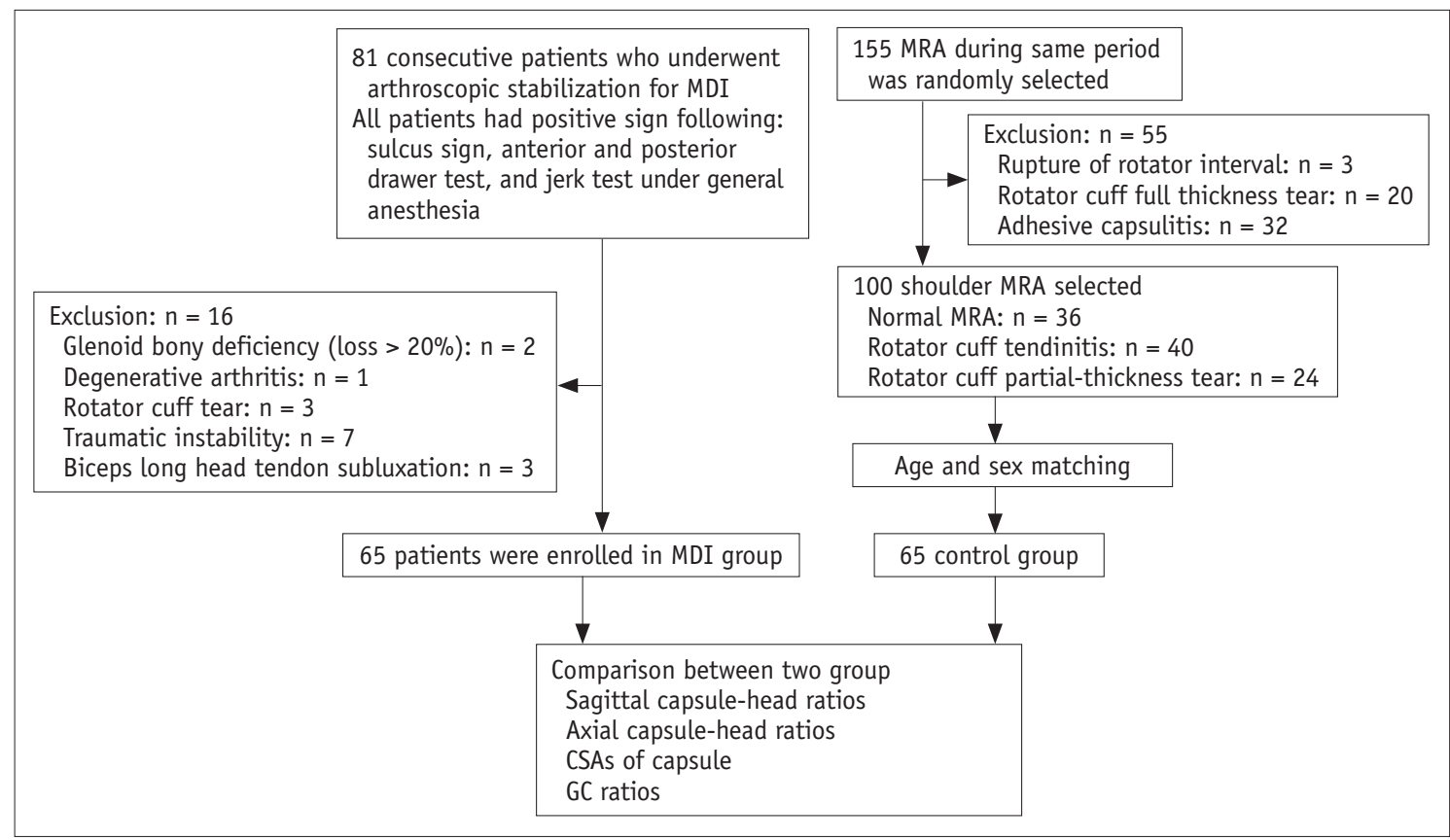

Fig. 1. Flow chart of study groups. $C S A s=$ cross-sectional areas, $G C=$ glenocapsular, $M D I=$ multidirectional instability, $M R A=$ magnetic resonance arthrography 
tendon (14 shoulders) without any limitations in the range of motion. We excluded patients who had any shoulder condition in which the intra-articular volume or pressure may have changed as a result of shoulder pathology, such as rotator cuff tendinitis and partial-thickness rotator cuff tears with limited range of motion, full-thickness rotator cuff tears, ruptures of the rotator interval, and adhesive capsulitis. After matching for age and sex, 65 control subjects ( 57 men, 8 women; mean age, 27.4 years; age range, $18-45$ years) were enrolled in the present study.

\section{MRA Protocol}

The 65 MDI patients underwent shoulder MRA prior to surgery. Approximately 15 minutes before MRI, each patient underwent shoulder arthrography. Briefly, under fluoroscopic guidance, a 22-gauge spinal needle was placed into the glenohumeral joint through the rotator interval. All patients were administered an intra-articular contrast mixture (injection volume, $10-16 \mathrm{~mL}$ ) until injection was no longer possible due to pressure, the plunger rebounded, or the patient complained of pain during the procedure. Injections were performed by an experienced musculoskeletal radiologist.

The contrast mixture was prepared by combining 0.1 $\mathrm{mL}$ of gadobutrol (Gadovist; Bayer AG, Berlin, Germany), $5 \mathrm{~mL}$ of iopamidol (Iopamiro; Bracco, Milan, Italy), $5 \mathrm{~mL}$ of $1 \%$ lidocaine, and $15 \mathrm{~mL}$ of saline. MRA images were obtained within 30 minutes of contrast injection using a 3T MR unit (Achieva TX; Philips Medical System, Eindhoven, The Netherlands) and a dedicated shoulder array. Patients underwent imaging in the supine position, with the examined arm alongside the body in slight external shoulder rotation with the palm facing upward. The following MRA imaging sequences were obtained: axial, oblique coronal, oblique sagittal T1-weighted sequences with fat saturation (axial repetition time [TR]/ echo time [TE] = $578.6 \mathrm{msec} / 10.1 \mathrm{msec}$, oblique coronal TR/TE $=868.8$ $\mathrm{msec} / 9.2 \mathrm{msec}$, oblique sagittal TR/TE $=4000 \mathrm{msec} / 30$ $\mathrm{msec}$ ) and axial, oblique coronal, and oblique sagittal T2weighted sequences $(\mathrm{TR} / \mathrm{TE}=3489 \mathrm{msec} / 80 \mathrm{msec})$. Oblique sagittal images were obtained parallel to the glenoid fossa. The sequence parameters used were as follows: section thickness, $3 \mathrm{~mm}$; interslice gap, $0.3 \mathrm{~mm}$; matrix, 256 x 192; and a field of view, $12 \times 12 \mathrm{~cm}$.

\section{GC Ratio Measurements}

Two orthopedic surgeons independently performed the
MRA analysis in a randomized blind fashion. Measurements were performed on MRA images using a picture archiving and communication system (PACS; Maroview version 5.4, MAROTECH Inc., Seoul, Korea).

The $\mathrm{GC}$ ratio was determined in each case by taking measurements from the oblique coronal fat-suppressed T1weighted image containing the largest glenoid cut and the insertion of the biceps long head. Distances from the superior aspect of the glenoid to the most inferior aspect of the capsule (A, Fig. 2), as well as those from the superior aspect of the glenoid to the most inferior aspect of the glenoid were recorded (B, Fig. 2). GC ratios were then calculated by dividing $A$ by $B$ in Figure 2 .

\section{Other Capsular Measurements}

In each case, on the axial image containing largest posterior capsular area and the glenoid, the distances between the anterior aspect of the lesser tuberosity and the most posterior aspect of the capsule (A, Fig. 3), as well as those from the anterior aspect of the lesser tuberosity to the posterior aspect of the humeral head along the line $(A$, Fig. 3) were taken ( $B$, Fig. 3). The axial capsule-head ratio was then calculated by dividing A by B in Figure 3 (14).

The sagittal capsule-head ratio was calculated by dividing the distance between the posterior aspect of the coracoid and the posterior humeral head (A, Fig. 4) by the distance between the posterior aspect of the coracoid and the most posterior aspect of the capsule (B, Fig. 4 ) on the sagittaloblique cut containing the largest posterior fluid pocket area (14).

In order to measure the sagittal cross-sectional area (CSA), the sagittal-oblique cut with the largest area of capsular pooling at or lateral to the glenoid rim was selected, and the total capsular area was recorded. On the same cut, the total area of the humerus was recorded. CSA was calculated by subtracting the total area of the humerus from the total area of the capsule on the same cut (Fig. 5) (14). All measurements were performed on the PACS with an electronic caliper.

\section{Statistical Analysis}

The capsular parameters GC ratio, axial capsule-head ratio, sagittal capsule-head ratio, and sagittal CSA of the MDI and control groups were compared using the Student's $t$ test. The mean values of the two observers were considered to be the final values. Of these four parameters, only those that differed significantly in the two groups were used. Cut-off 


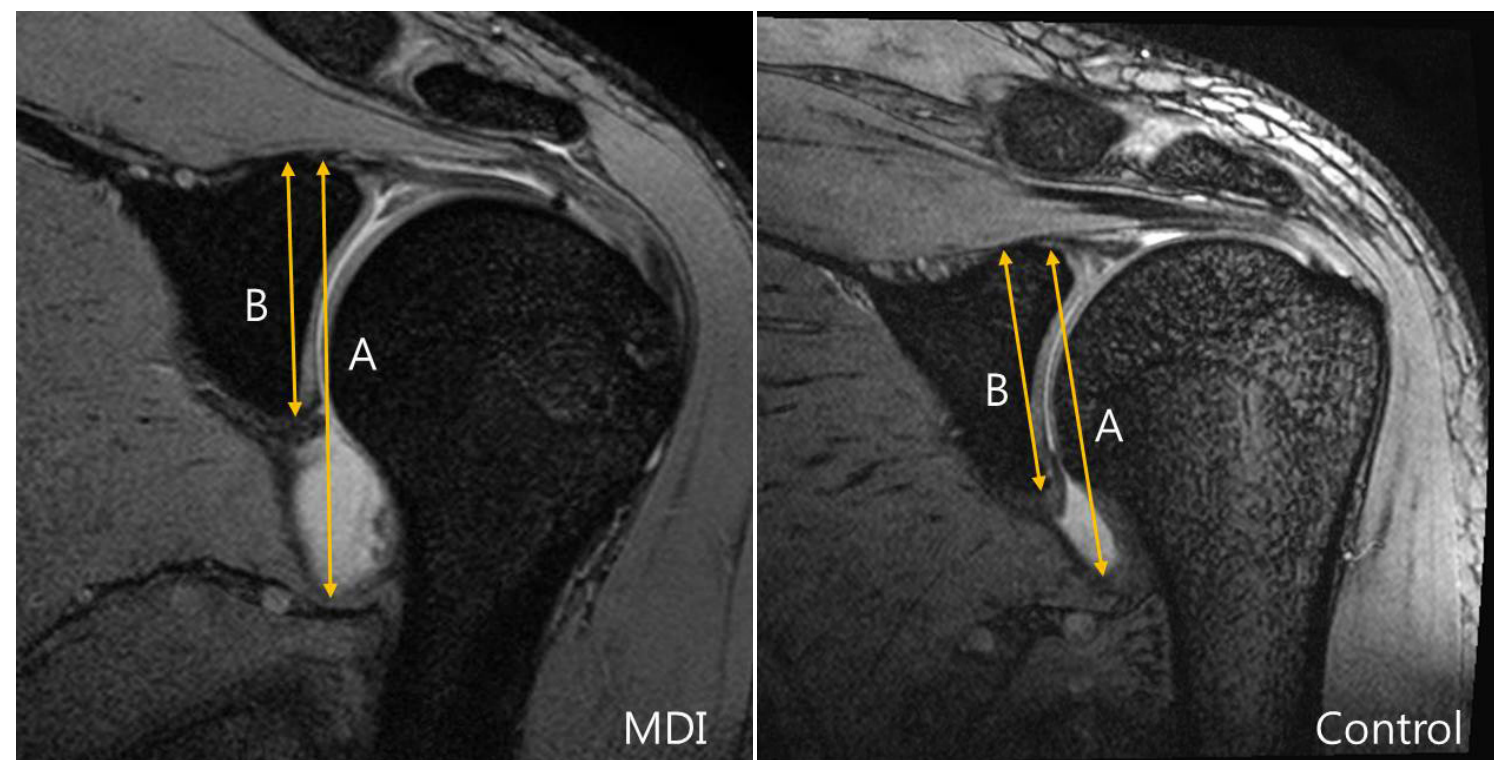

Fig. 2. GC ratios were obtained on oblique coronal images showing largest glenoid cut and insertion of biceps long head. Line A was drawn from top of glenoid to bottom of inferior capsule. Line B was drawn from top of glenoid to bottom of glenoid parallel to line A. GC ratio was calculated by dividing length of $A$ by that of $B$.

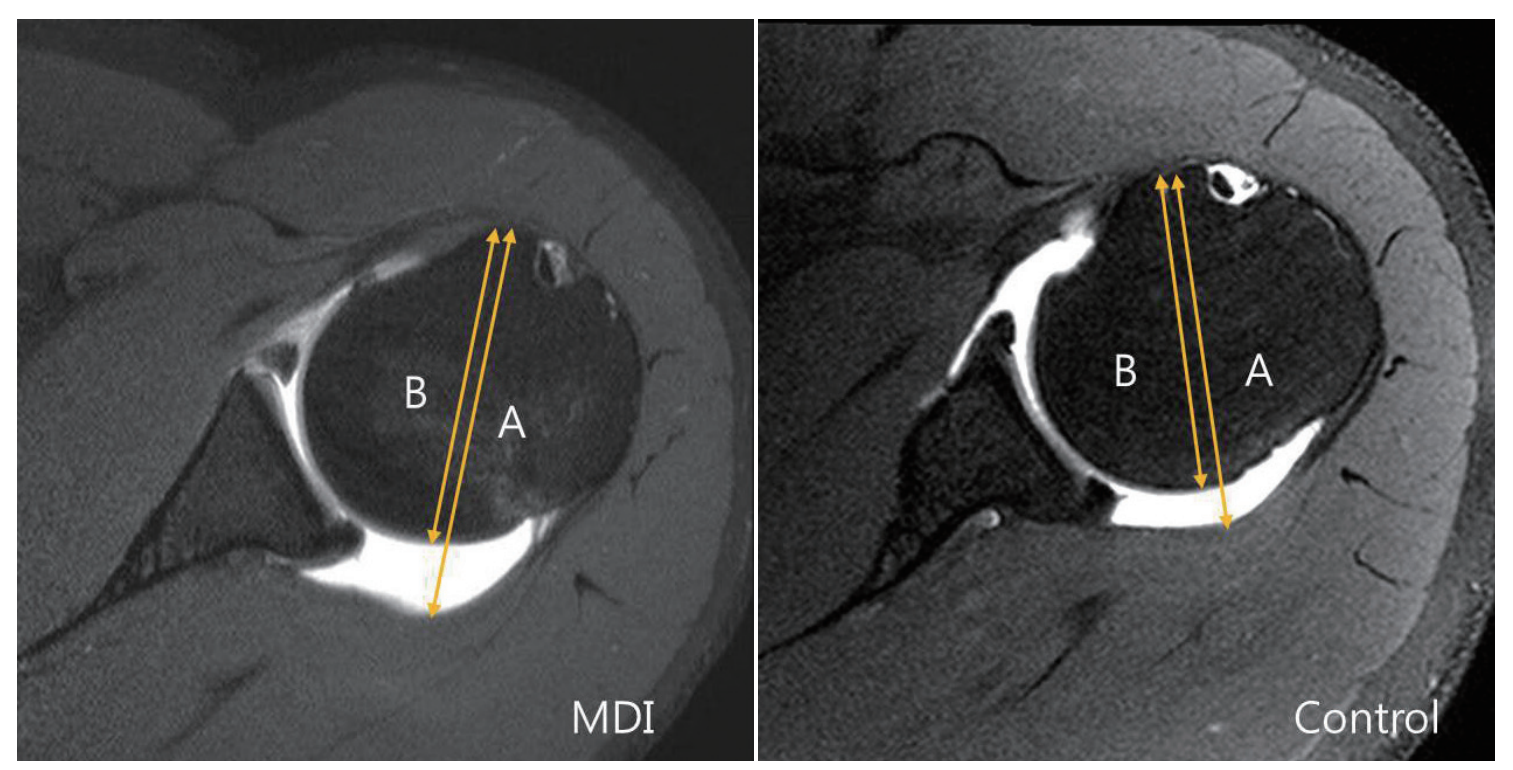

Fig. 3. Axial capsule-head ratios were obtained using axial images showing largest posterior capsular area and glenoid. Line A was drawn from anterior aspect of lesser tuberosity to most posterior aspect of posterior capsule. Line B was drawn from anterior aspect of lesser tuberosity to posterior aspect of humeral head parallel to line A. Axial capsule-head ratio was calculated by dividing length of line A by that of B.

values for predicting MDI of the shoulder were determined by receiver operating characteristic (ROC) curve analysis. Interobserver agreement between the two orthopedic surgeons was quantified using Pearson's correlation coefficient (Pearson's $r$ ). All statistical tests were performed with SPSS for Windows version 17.0 (SPSS Inc., Chicago, IL, USA). Statistical significance was accepted for $p$ values < 0.05 .

\section{RESULTS}

The interobserver reliability was very good for the measurements of GC ratio, sagittal capsule-head ratio, and axial capsule-head ratio (correlation coefficients, 0.94, 0.82, and 0.95 , respectively), and good for CSA measurements (correlation coefficient, 0.79).

$\mathrm{GC}$ ratios were significantly larger in the patient group than in the control group (control group, $1.33 \pm 0.07$; MDI 

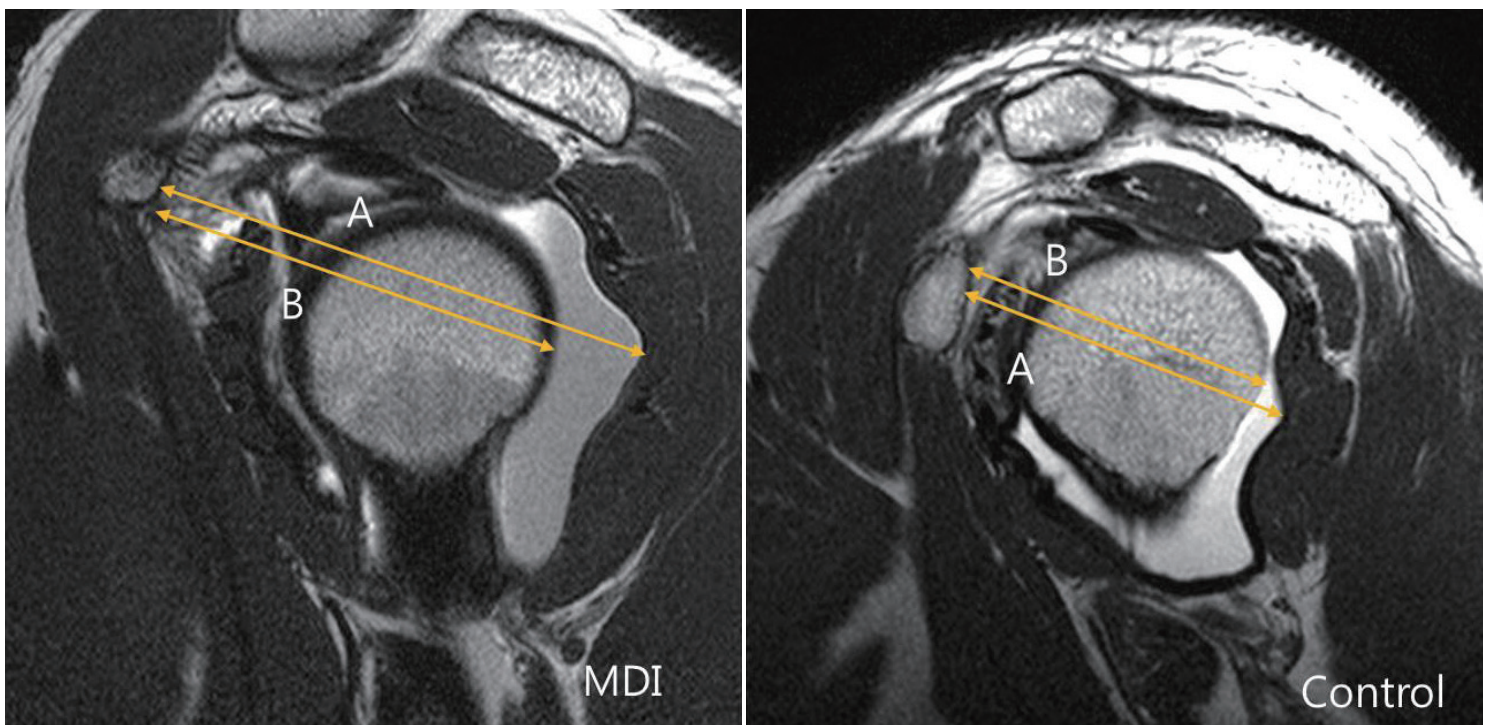

Fig. 4. Sagittal capsule-head ratios were obtained using sagittal-oblique images showing largest posterior fluid pocket. Line A was drawn from posterior aspect of coracoid to most posterior aspect of capsule, and line B from posterior aspect of coracoid to posterior aspect of humeral head parallel to line A. Sagittal capsule-head ratio was calculated by dividing length of line A by that of line B.
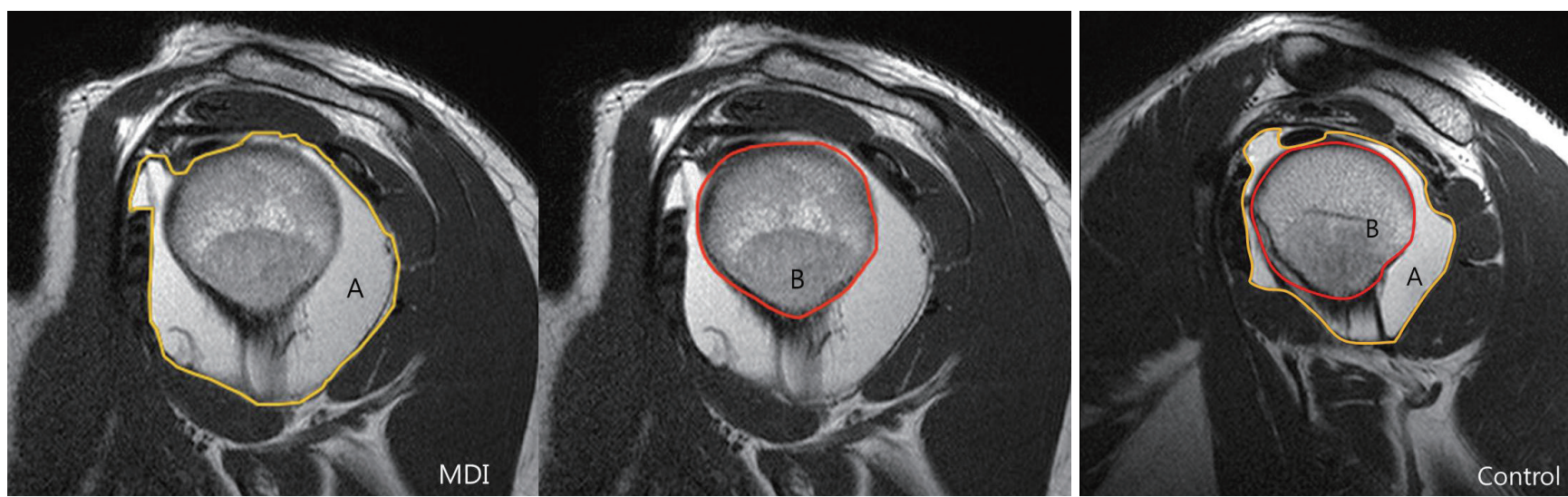

Fig. 5. Measurement of sagittal CSA. To measure sagittal CSA, sagittal-oblique cut with largest area of capsular pooling at or lateral to glenoid rim was selected, and total area of capsule was recorded A. On same cut, total area of humerus was recorded B. CSA was calculated by subtracting total area of humerus from total capsule area on same cut.

group, $1.49 \pm 0.08, p<0.001)$. Mean CSA was significantly larger in patients as well (control group, $523.9 \pm 240.9$; MDI group, $964.8 \pm 280.8, p<0.001)$. However, sagittal capsule-head ratios were not significantly different between the patient and control groups (control group, $1.12 \pm 0.03$; MDI group, $1.18 \pm 0.16, p=0.317$ ), and axial capsule-head ratios were not significantly different either (control group, $1.10 \pm 0.03$; MDI group, $1.18 \pm 0.08, p=0.053$ ) (Table 1).

The calculated area under the ROC curve for $\mathrm{GC}$ ratio was 0.925 . For CSA, the area under the curve was 0.891 (Fig. 6). For a threshold GC ratio of 1.45 , the sensitivity and specificity for the detection of MDI were $86.2 \%$ and $93.8 \%$, respectively, and for a GC ratio of 1.42 , the sensitivity and specificity were $92.3 \%$ and $89.2 \%$, respectively (Supplementary Table 1 in the online-only Data Supplement). For a threshold CSA of $785 \mathrm{~mm}^{2}$, the sensitivity and specificity for the detection of MDI were $73.8 \%$ and $92.3 \%$, respectively, and for a threshold CSA of $699 \mathrm{~mm}^{2}$, the sensitivity and specificity were $86.2 \%$ and $73.8 \%$, respectively (Supplementary Table 1 in the onlineonly Data Supplement).

\section{DISCUSSION}

In the present study, CSAs and GC ratios were found to be significantly greater in clinically diagnosed MDI patients than in control subjects with no instability. In addition, the study indicates that MDI of the shoulder should be 
Table 1. Measurements Obtained in Patient and Control Groups

\begin{tabular}{llcc}
\hline \multicolumn{1}{c}{ Measurements } & Group & $\begin{array}{c}\text { Average } \\
( \pm \text { Standard Deviation })\end{array}$ & $P$ \\
\hline GC ratio & MDI & $1.49 \pm 0.08$ & $<0.001^{*}$ \\
Axial capsule-head & Control & $1.33 \pm 0.07$ & \\
ratio & MDI & $1.18 \pm 0.08$ & 0.053 \\
Sagittal head-capsule & Control & $1.10 \pm 0.03$ & \\
ratio & MDI & $1.18 \pm 0.16$ & 0.317 \\
CSA & Control & $1.12 \pm 0.03$ & \\
& MDI & $964.8 \pm 280.8$ & \\
& Control & $523.9 \pm 240.9$ & $<0.001^{*}$ \\
\hline
\end{tabular}

${ }^{*} p<0.05$ indicative of significance. CSA $=$ cross-sectional area, GC = glenocapsular, MDI = multidirectional instability of shoulder

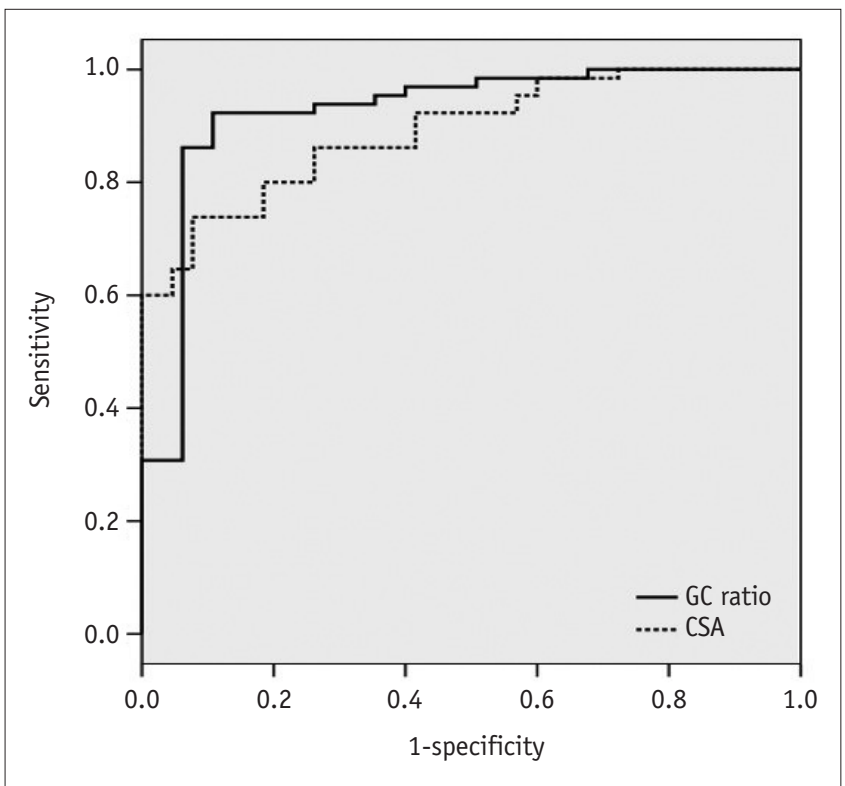

Fig. 6. ROC curves for GC ratio and CSA. Threshold values and diagnostic sensitivities and specificities were calculated using ROC curves. Mean GC ratio and CSA were significantly different in multidirectional shoulder instability group and control group. $\mathrm{ROC}=$ receiver operating characteristic

suspected in patients with $\mathrm{GC}$ ratios $>1.42$. Furthermore, compared to CSA, GC ratio was found to predict the presence of MDI of the shoulder more accurately.

The main contributors to capsular redundancy in MDI are an elongated inferior capsule and a deficient rotator interval (9-11). Inferior instability of the shoulder is the main component of MDI, and distal migration or inferior translation of the humerus suggests MDI (15). Previous studies have reported that the widths, depths, and areas of the rotator intervals were greater in MDI patients than in controls $(11,12)$. In patients with MDI, correction of the inferior capsular redundancy has become the fundamental component of surgical treatment. In previous studies, inferior capsular plication was used to reduce the range of motion to the intact state $(3,16,17)$, although capsular plication with rotator interval closure has been used as well.

The studies mentioned above reported that inferior capsular laxity was a significant pathological condition in MDI and that inferior capsular shift was important for treatment. Several studies on MDI have focused on the inferior capsule on MRA images. For example, Dewing et al. (14) performed capsular area analysis of shoulder instability and reported an increase in inferior capsule CSA in patients with posterior or MDI, as compared to controls. In that study, anteroinferior and posteroinferior (PI) CSAs were measured on sagittal-oblique images with the most distended capsular area by bisecting the humeral head along a line down the center of the humeral shaft. The results indicated that PI capsular areas were greater in the shoulders of MDI patients than in those of controls. Lee et al. (11) reported that capsular dimensions in the inferior and PI regions were significantly larger in an MDI group than in controls, and Lim et al. (13) reported that inferior capsule lengths were larger in MDI patients than in controls. These findings are consistent with those of the present study. In previous studies, as in the present study, redundancy of the inferior capsule was reported to be important, and all of the studies compared redundancies with controls. Dewing et al. (14) reported that mean sagittal CSA was $11.4 \mathrm{~cm}^{2}$ in MDI patients and $7.7 \mathrm{~cm}^{2}$ in controls, whereas we found that mean sagittal CSA was 9.64 $\mathrm{cm}^{2}$ in patients and $5.23 \mathrm{~cm}^{2}$ in controls; these differences were significant in both studies. However, the CSA values differed, and mean sagittal CSAs in the present study were smaller than those in Dewing's study, which may be due to differences in the sizes of the joint structures in the two studies. In a study by Lim et al. (13), it was proposed that a threshold inferior capsule length of $16.88 \mathrm{~mm}$ can be used for MDI screening with a sensitivity of $76 \%$ and a specificity of $96 \%$. However, this study was undertaken in a Korean cohort, and different results, perhaps larger values in particular, would be expected in Caucasians due to the typically larger skeletons. In the present study, GC ratio was defined as inferior capsule length divided by glenoid length in order to compensate for patient size differences, and this ratio had a sensitivity of $86.2 \%$ and a specificity of $93.8 \%$ when a threshold GC ratio of 1.45 was applied, which suggests that the $\mathrm{GC}$ ratio could be used as a universal screening parameter for MDI. 
Our study had several limitations. First, although MRA with contrast injection is an established technique that was performed in a standardized fashion, the techniques used likely vary in terms of the location of the injection (1820). However, fluoroscopic guidance and the standardized method of performing the injection may have reduced concerns regarding inadequate fluid injection. Second, the volumes of joint capsules on MRA images and amounts of contrast medium injected into the glenohumeral joint varied. We injected diluted gadolinium using gentle pressure until injection was no longer possible due to the increased intra-articular pressure, which is believed to provide a reasonable means of assessing individual capsular volumes, but injection pressures were not measured, as was the case in previous studies (11-14). However, all MRA injections were performed by one experienced musculoskeletal radiologist, and that could have reduced the variation in the amount of injected contrast material. In future MRA studies, we suggest that injection pressures be measured so as to ensure that intra-articular injections are made consistently. Third, our cohort showed a male predominance. In order to account for this, we matched the patient and control groups for sex and age.

The strength of our study is that MDI patients were rigorously selected. MDI was confirmed in all cases by arthroscopic surgery and physical examination under general anesthesia. Furthermore, the linear measures used were computer-derived, easily obtainable, and reproducible and had high interobserver correlations.

The present study demonstrates the existence of a strong relationship between atraumatic MDI of shoulder and elongation of the inferior capsule as determined with MRA-derived measures. We recommend that the $\mathrm{GC}$ ratio be considered as the basis of a new screening method for atraumatic MDI of the shoulder. Furthermore, our findings provide further biomechanical and surgical evidence that inferior capsular size plays a role in revealing the pathogenesis of MDI of the shoulder.

\section{Supplementary Materials}

The online-only Data Supplement is available with this article at https://doi.org/10.3348/kjr.2018.0541.

\section{Conflicts of Interest}

The authors have no potential conflicts of interest to disclose.
Acknowledgments

I extend my appreciation to all colleagues that aided me during this study.

\author{
ORCID iDs \\ Ho-Seung Jeong \\ https://orcid.org/0000-0001-7297-5534 \\ Kyoung-Jin Park \\ https://orcid.org/0000-0001-9373-1440
}

\section{REFERENCES}

1. Neer CS 2nd, Foster CR. Inferior capsular shift for involuntary inferior and multidirectional instability of the shoulder. A preliminary report. J Bone Joint Surg Am 1980;62:897-908

2. Gerber C, Nyffeler RW. Classification of glenohumeral joint instability. Clin Orthop Relat Res 2002;(400):65-76

3. Choi CH, Ogilvie-Harris DJ. Inferior capsular shift operation for multidirectional instability of the shoulder in players of contact sports. Br J Sports Med 2002;36:290-294

4. Misamore GW, Sallay PI, Didelot W. A longitudinal study of patients with multidirectional instability of the shoulder with seven- to ten-year follow-up. J Shoulder Elbow Surg 2005; $14: 466-470$

5. Pollock RG, Owens JM, Flatow EL, Bigliani LU. Operative results of the inferior capsular shift procedure for multidirectional instability of the shoulder. J Bone Joint Surg Am 2000;82-A:919-928

6. McIntyre LF, Caspari RB, Savoie FH 3rd. The arthroscopic treatment of multidirectional shoulder instability: twoyear results of a multiple suture technique. Arthroscopy 1997; 13:418-425

7. Johnson SM, Robinson CM. Shoulder instability in patients with joint hyperlaxity. J Bone Joint Surg Am 2010;92:15451557

8. Levine WN, Prickett WD, Prymka M, Yamaguchi K. Treatment of the athlete with multidirectional shoulder instability. Orthop Clin North Am 2001;32:475-484

9. Barden JM, Balyk R, Raso VJ, Moreau M, Bagnall K. Atypical shoulder muscle activation in multidirectional instability. Clin Neurophysiol 2005;116:1846-1857

10. Hsu YC, Pan RY, Shih YY, Lee MS, Huang GS. Superior-capsular elongation and its significance in atraumatic posteroinferior multidirectional shoulder instability in magnetic resonance arthrography. Acta Radiol 2010;51:302-308

11. Lee HJ, Kim NR, Moon SG, Ko SM, Park JY. Multidirectional instability of the shoulder: rotator interval dimension and capsular laxity evaluation using MR arthrography. Skeletal Radiol 2013;42:231-238

12. Kim KC, Rhee KJ, Shin HD, Kim YM. Estimating the dimensions of the rotator interval with use of magnetic resonance arthrography. J Bone Joint Surg Am 2007;89:2450-2455 
13. Lim CO, Park KJ, Cho BK, Kim YM, Chun KA. A new screening method for multidirectional shoulder instability on magnetic resonance arthrography: labro-capsular distance. Skeletal Radiol 2016;45:921-927

14. Dewing CB, McCormick F, Bell SJ, Solomon DJ, Stanley M, Rooney TB, et al. An analysis of capsular area in patients with anterior, posterior, and multidirectional shoulder instability. Am J Sports Med 2008;36:515-522

15. Helmig P, Søjbjerg J0, Kjaersgaard-Andersen P, Nielsen $\mathrm{S}$, Ovesen J. Distal humeral migration as a component of multidirectional shoulder instability. An anatomical study in autopsy specimens. Clin Orthop Relat Res 1990;(252):139-143

16. Kim SH, Kim HK, Sun JI, Park JS, Oh I. Arthroscopic capsulolabroplasty for posteroinferior multidirectional instability of the shoulder. Am J Sports Med 2004;32:594-607

17. Shafer BL, Mihata T, McGarry MH, Tibone JE, Lee TQ. Effects of capsular plication and rotator interval closure in simulated multidirectional shoulder instability. J Bone Joint Surg Am 2008;90:136-144

18. Lubowitz J, Bartolozzi A, Rubinstein D, Ciccotti M, Schweitzer $M$, Nazarian $L$, et al. How much does inferior capsular shift reduce shoulder volume? Clin Orthop Relat Res 1996;(328):8690

19. Luke TA, Rovner AD, Karas SG, Hawkins RJ, Plancher KD. Volumetric change in the shoulder capsule after open inferior capsular shift versus arthroscopic thermal capsular shrinkage: a cadaveric model. J Shoulder Elbow Surg 2004;13:146-149

20. Resnik CS, Fronek J, Frey C, Gershuni D, Resnick D. Intraarticular pressure determination during glenohumeral joint arthrography. Preliminary investigation. Invest Radiol 1984;19:45-50 